Apidologie, 1978, 9 (1), 19-32.

\title{
VERGLEICHENDE MORPHOLOGIE DES STACHELAPPARATES BEI DEN VIER APIS-ARTEN (HYMENOPTERA : APIDAE)
}

\author{
Morphologie comparée de l'appareil venimeux \\ chez les quatre espèces d'Apis (Hymenoptera : Apidae) \\ Jürgen WEISS \\ Institut für Bienenkunde, 637 Oberursel, Im Rotkopf 5 \\ SUMMARY \\ COMPARATIVE MORPHOLOGY OF THE STING APPARATUS \\ IN THE GENUS APIS (HYMENOPTERA : APIDAE)
}

1. From an interspecific comparison of the morphology of the sting apparatus in the genus Apis (Apis dorsata FABricius, 1793, A. mellifera Linnaeus, 1758, A. cerana FABricius, 1793, A. florea FABRICIUS, 1787) resulted that most of the structures are related in their sizes to the exterior proportions of the respective species. The highly specialized barbs of stilet and lancets showed a diverging situation.

2. Stilet barbs : four pairs of well developed barbs in A. florea, three to four in A. dorsata and A. cerana. A. mellifera normally does not have barbs with a measurable posterior depth of notches.

3. Lancet barbs : each lancet of $A$. dorsata, A. mellifera and $A$. cerana is equipped with eleven barbs, in A. florea with ten only. The first three lancet barbs of $A$. dorsata and $A$. mellifera are well developed, those of A. cerana are extremely weak.

4. The ninth lancet barb : the angle between the ninth barb and the longitudinal axis of the lancet is of this sequence : A. dorsata $\left(20^{\circ}\right)$, A. florea $\left(18^{\circ}\right)$, A. mellifera $\left(10^{\circ}\right)$ and $A$. cerana $\left(7^{\circ}\right)$. The posterior depth of the notch of the ninth lancet barb is : A. dorsata $(22,95 \mu \mathrm{m})$, A. mellifera $(16,75 \mu \mathrm{m})$, A. florea $(14,62 \mu \mathrm{m})$ and A.cerana $(10,13 \mu \mathrm{m})$.

5. In regard to the exterior proportions of the four species $A$. florea has the best developed barbs of the genus Apis, followed by A.dorsata and A. mellifera; but A.cerana seems to be the most primitive species in the respects mentioned above.

\section{ZUSAMMENFASSUNG}

1. Ein interspezifischer Vergleich der Stachelmorphologie der vier Apis-Arten (Apis dorsata Fabricius, 1793, A. mellifica Linnaeus, 1758, A. cerana Fabricius, 1793. A. forea Fabricius, 1787) ergab, dass die meisten Strukturen in ihren Proportionen den äusseren Grössenverhältnissen der Arten entsprechen. Die spezialisierten Widerhakenstrukturen von Stachelrinne und Stechborsten dagegen zigisn ein abweichendes Bild. 
2. Widerhaken der Stachelrinne : mit vier Paar gut entwickelter Widerhaken liegt $A$. florea an der Spitze, es folgen $A$. dorsata und A. cerana; $A$. mellifica weist in der Regel keine Widerhaken mit messbarem posteriorem Einschnitt auf.

3. Widerhaken der Stechborsten : A. dorsata, A. mellifica und A. cerana haben elf Widerhaken je Stechborste, A. florea nur zehn.

Bei den Stechborsten von A. dorsata, A. mellifica und A. florea sind die ersten drei Widerhaken gut ausgebildet, bei $A$. cerana sind diese Strukturen extrem schwach entwickelt.

4. IX. Stechborstenwiderhaken: Bezogen auf den Winkel zwischen Widerhaken und Stechborstenlängsachse ergibt sich folgende Reihenfolge : A. dorsata: $20^{\circ}(\mathrm{s}=1,49)$, A.florea: $18^{\circ}$ (s=1,15), A. mellifica: $10^{\circ}(\mathrm{s}=0,94)$ und $A$. cerana : $7^{\circ}(\mathrm{s}=0,94)$.

Nach der posterioren Einschnittiefe ergibt sich diese Reihung: A. dorsata: $22,95 \mu \mathrm{m}(\mathrm{s}=1,52)$, A. mellifica : $16,75 \mu \mathrm{m}(\mathrm{s}=0,64)$, A. florea : $14,62 \mu \mathrm{m}(\mathrm{s}=0,7)$ und A. cerana $: 10,13 \mu \mathrm{m}(\mathrm{s}=0,31)$.

5. Berücksichtigt man bei den oben aufgeführten Befunden die Proportionsunterschiede der vier Arten, so sind nach allen untersuchten Kriterien die Widerhakenstrukturen von $A$.florea am besten entwickelt, es folgen $A$. dorsata und $A$. mellifica; $A$. cerana erscheint am schwächsten entwickelt und damit am ursprünglichsten.

\section{I. - EINLEITUNG}

Die Morphologie des Stachelapparates von A. mellifica kann heute als gut untersucht gelten (ZaNDER, 1951; SNOdgrass, 1956 u. a.). Neben wenigen vergleichenden Untersuchungen an Stachelapparaten von A. mellifica und A. cerana (SAKagami und AKahIRA, 1960; BäHRMANN, 1967) fehlt ein interspezifischer Vergleich aller vier Arten - insbesondere unter Berücksichtigung der beiden freibrütenden Arten - bisher. Ein solcher Vergleich soll Gegenstand der vorliegenden Untersuchung sein.

\section{II. - MATERIAL UND METHODEN}

Für die Untersuchungen am Rasterelektronenmikroskop wurden die Stachelapparate von jeweils zehn Flugbienen der vier $A$ pis-Arten ausgewertet. Die Tiere von $A$. mellifica gehörten der carnica-Rasse an, die Exemplare der drei asiatischen Arten stammten aus Sri Lanka (Anuradhapura und Kandy).

Besondere Fixiermethoden waren wegen der ausschliesslich chitinösen Beschaffenheit der hier besprochenen Strukturen nicht erforderlich. Verwendet wurde ein Rasterelektronenmikroskop vom Typ S 500 der Firma HITACHI.

$$
\text { III. - ERGEBNISSE }
$$

Der Stachelapparat.

Der Stachelapparat der Honigbienen - der sich wie bei allen aculeaten Hymenopteren aus einem Legestachel entwickelt hat - ist in der grossen 
Stachelkammer am hinteren Abdominalende enthalten (Abb. 1) : Er besteht aus drei Paaren von Chitinplatten (motorischer Apparat) als dem basalen Teil, dem spitz auslaufenden Stachelschaft, sowie den Drüsen des Stachelapparates.

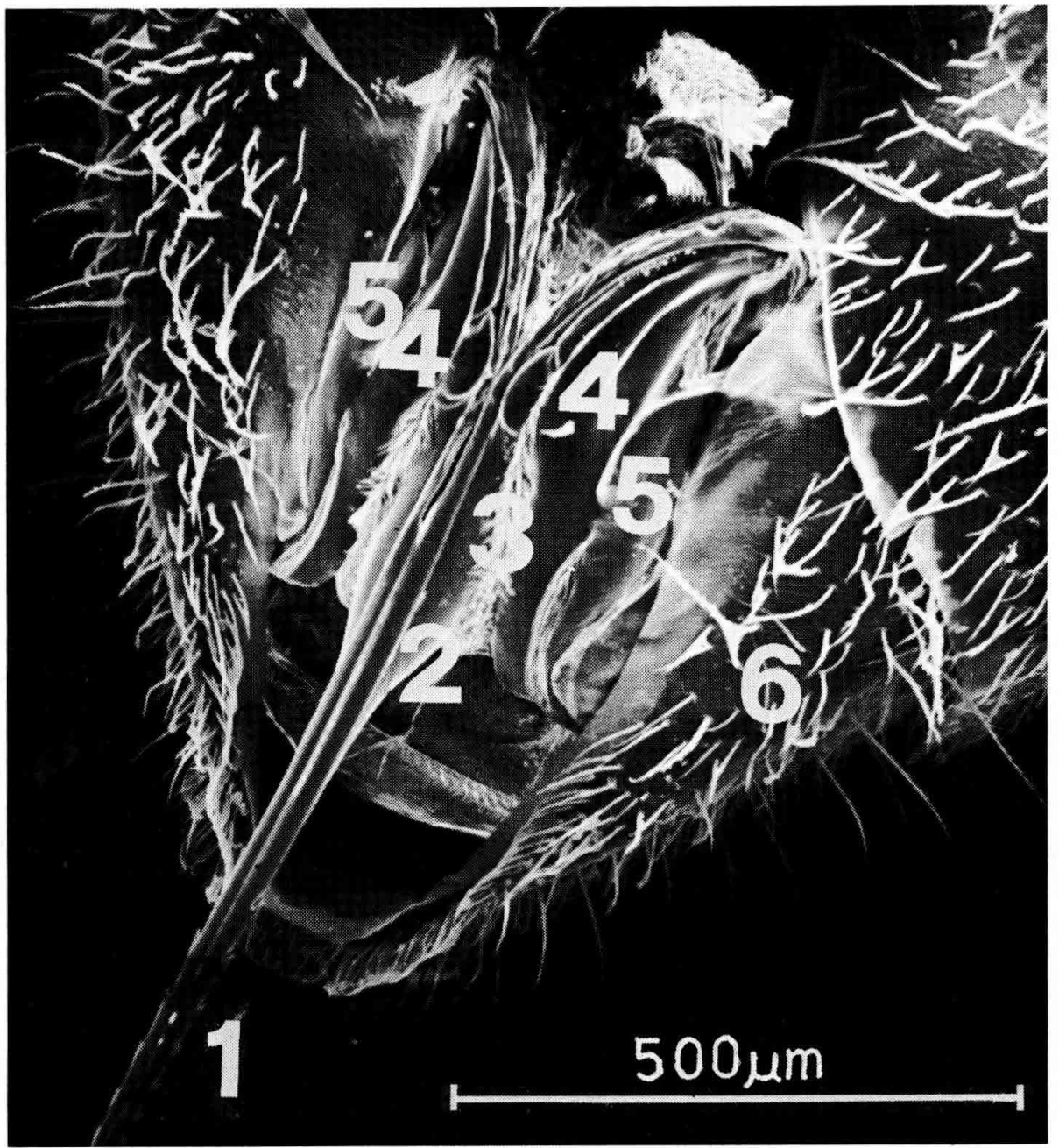

AвB. 1. - Ventraler Stachelsitus einer Arbeiterin von Apis dorsata. Die VI.

Sternalplatte wurde entfernt (REM) :

1. Stechborsten. 2. Bulbus. 3. Stachelrinnenpolster. 4. Oblonge Platte.

5. Quadratische Platte. 6. Tergalplatte VI.

FIG. 1. - Vue ventrale de l'aiguillon d'une ouvrière d'Apis dorsata. La $6^{\mathbf{e}}$ plaque sternale a été enlevée

(microscopie électronique à balayage)

1. Soie barbelée. 2. Bulbe. 3. Coussin des gaines de l'aiguillon.

4. Plaque oblongue. 5. Plaque angulaire. 6. Sixième plaque tergale. 
Die Plattenpaare des motorischen Apparates - quadratische und oblonge Platten - sind gelenkig miteinander und über die Winkel mit den Stechborstenbögen verbunden. Letztere gehen posterior im Bereich des Stachelschaftes in die starren, geraden Stechborsten über. An der dorsalen Basis des Stachelschaftes mündet der Bulbus posterior in die Stachelrinne. Diese trägt auf ihren ventralen, lateralen Rändern schienenartige Leisten, auf denen die Stechborsten mit ihren - analog geformten Furchen laufen. Die posterioren Enden des (ventralen) Stechborstenpaares bilden gemeinsam mit dem Ende der unpaaren Stachelrinne die Stachelspitze. Die Stachelrinne weist dorsal im Bereich ihrer Spitze und die Stechborsten auf ihren lateralen Aussenkanten unterschiedlich gut ausgebildete Widerhakenstrukturen auf.

Der interspezifische Vergleich der Stachelmorphologie ergab, dass der grösste Teil der untersuchten Strukturen in seinen Proportionen den äusseren Grössenverhältnissen der einzelnen Arten entspricht. Form und Grösse der Widerhakenstrukturen an Stachelrinne und Stechborsten haben jedoch ein abweichendes Bild ergeben. Ihr interspezifischer Vergleich soll in der Folge beschrieben werden.

\section{A. - Widerhaken der Stachelrinne}

Der interspezifische Vergleich zeigte, dass die Stachelrinne aller untersuchten Stachelapparate von A. florea vier Paar gut ausgebildeter Widerhaken mit messbarem posteriorem Einschnitt besitzt (Abb. 2). A. mellifica dagegen weist nur vereinzelt Ansätze widerhakenähnlicher Strukturen auf (siehe dazu auch SAKagami und AKAHIRA, 1960), die jedoch in der Regel nicht posterior eingeschnitten sind (Abb. 3).

Während bei $A$. cerana das vierte Widerhakenpaar der Stachelrinne meist stark reduziert oder überhaupt nicht ausgebildet ist, weist $A$. dorsata immerhin an vier von zehn untersuchten Stachelrinnen ein viertes Widerhakenpaar auf.

\section{B. - Widerhaken der Stechborsten}

Ein Vergleich der Widerhakenanzahl ergab, dass A. dorsata. A. mellifica und $A$. cerana elf Widerhaken je Stechborste besitzen, A. florea jedoch nur zehn (Abb. 4).

\section{B. 1. - Erster bis dritter Widerhaken}

Da Form und Grösse - besonders der vordersten - Stechborstenwiderhaken für das Festhaken des Stachelschaftes von Bedeutung sind, wurden zunächst die Widerhakenpaare eins bis drei vermessen (Abb. 5) : Während A. dorsata, A. mellifica und $A$. florea in allen untersuchten Fällen gut entwickelte erste, zweite und dritte Widerhakenpaare mit ausgeprägten posterioren Einschnitten besitzen, ist bei $A$. cerana das erste Widerhakenpaar in neun von zehn Fällen nur als schwache Ausbuchtung ohne messbaren Einschnitt vorhanden (Abb. 6). 

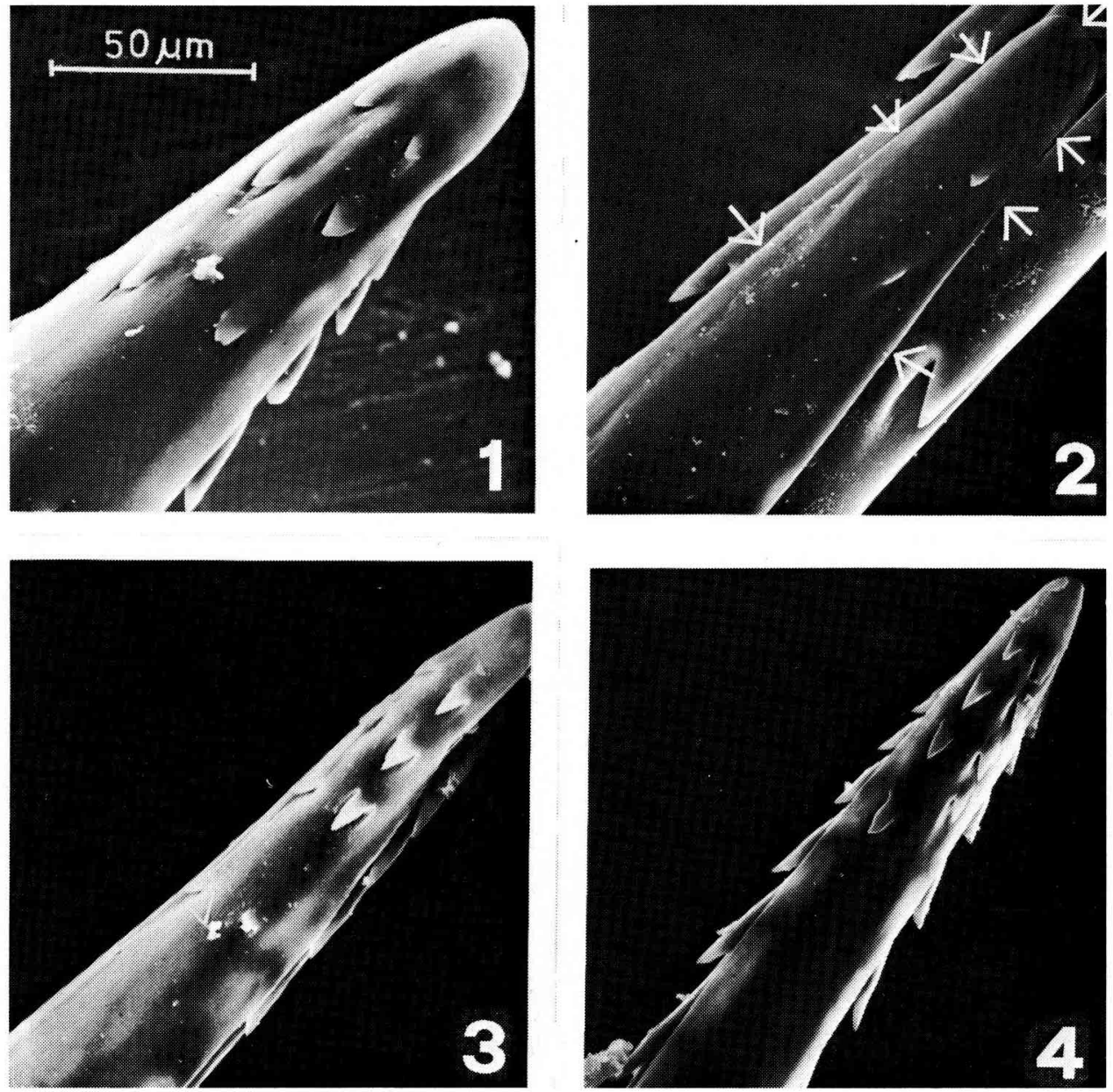

ABB. 2. - Dorsalansicht der Stachelrinnenspitze der vier Apis-Arten (REM).

Seitlich, bzw unter der Spitze der Stachelrinnen sind mehr oder weniger deutlich noch Teile der Stechborsten mit ihren Widerhaken zu erkennen.

Bei 2 ist der Umriss der Stachelrinne durch Pfeile hervorgehoben :

1. A. dorsata. 2. A. mellifica. 3. A. cerana. 4. A. florea.

FIG. 2. - Vue dorsale de la pointe des gaines de l'aiguillon des 4 espèces d'Apis (microscopie électronique à balayage).

Sur le côté ou sous la pointe des gaines de l'aiguillon on reconnaît plus ou moins nettement des fragments des soies barbelées avec leurs barbes. Sur la figure 2.2. le contour des gaines est souligné par des flèches.

2.1. A. dorsata. 2.2. A. mellifica. 2.3. A. cerana. 2.4. A. florea. 


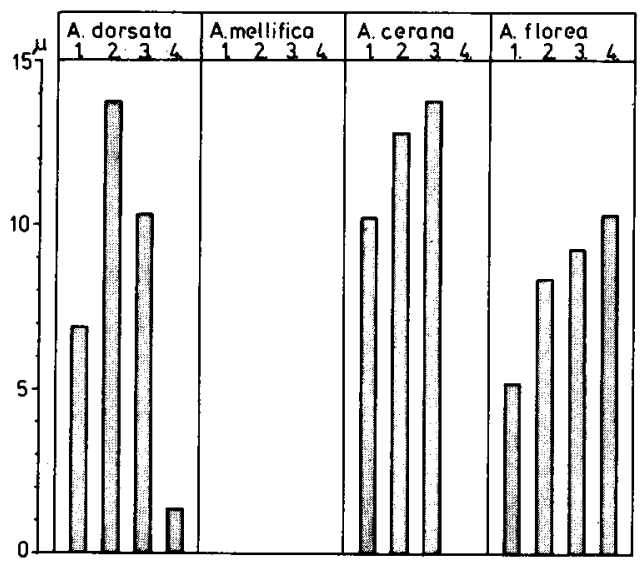

Aв8. 3. - Posteriore Einschnittiefen der Stachelrinnenwiderhaken bei den vier Apis-Arten (REM). Angaben in Mikrometer.

FIG. 3. - Profondeur des crans postérieurs des barbes des gaines de l'aiguillon chez les 4 espèces d'Apis (microscopie électronique à balayage).

Mesures en $\mu \mathrm{m}$.

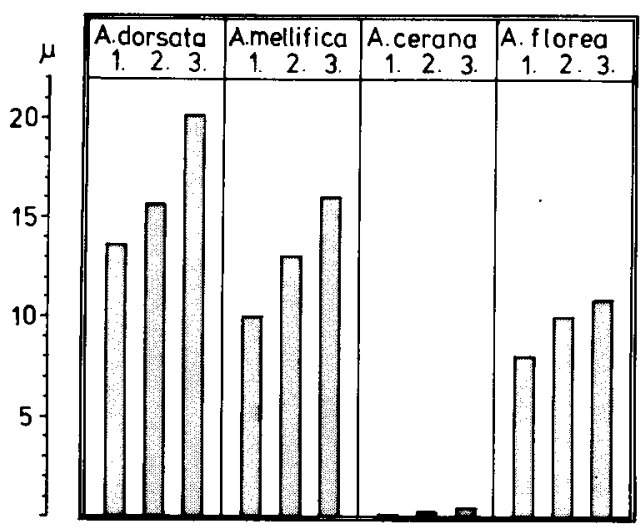

ABв. 5. - Posteriore Einschnittiefe der Stechborstenwiderhaken I bis III. Angaben in Mikrometer.

Fig. 5. - Profondeur des crans postérieurs des barbes I à III des soies barbelées. Mesures en $\mu \mathrm{m}$. 

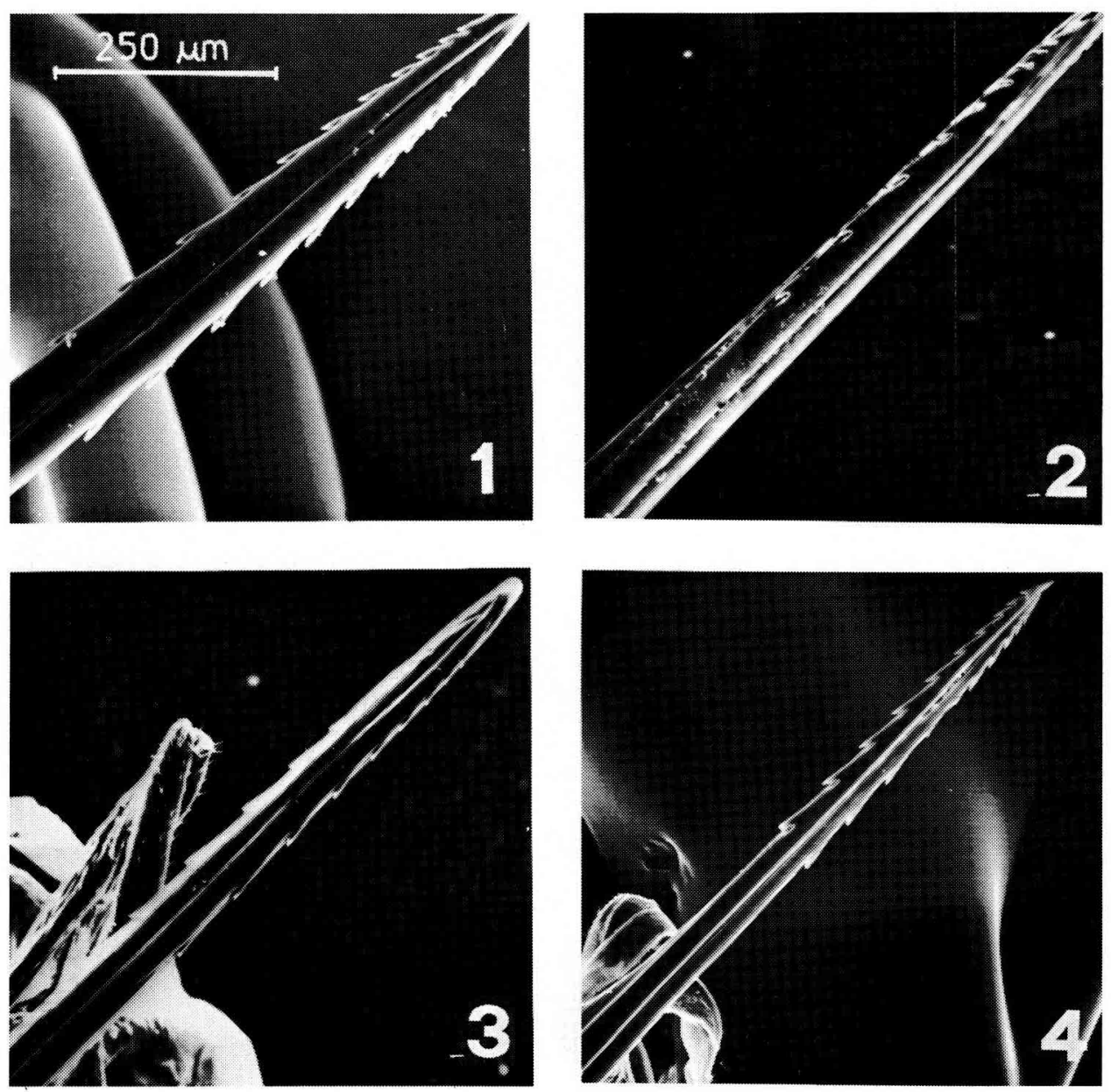

Авв. 4. - Stechborsten der vier Apis-Arten mit ihren Widerhaken (REM):

1. A. dorsata. 2. A. mellifica. 3. A. cerana. 4. A. florea.

FIG. 4. - Soles barbelées des 4 espèces d'Apis avec leurs barbes (microscopie électronique à balayage).

4.1. A. dorsata. 4.2. Apis mellifica. 4.3. A. cerana. 4.4. A. florea. 

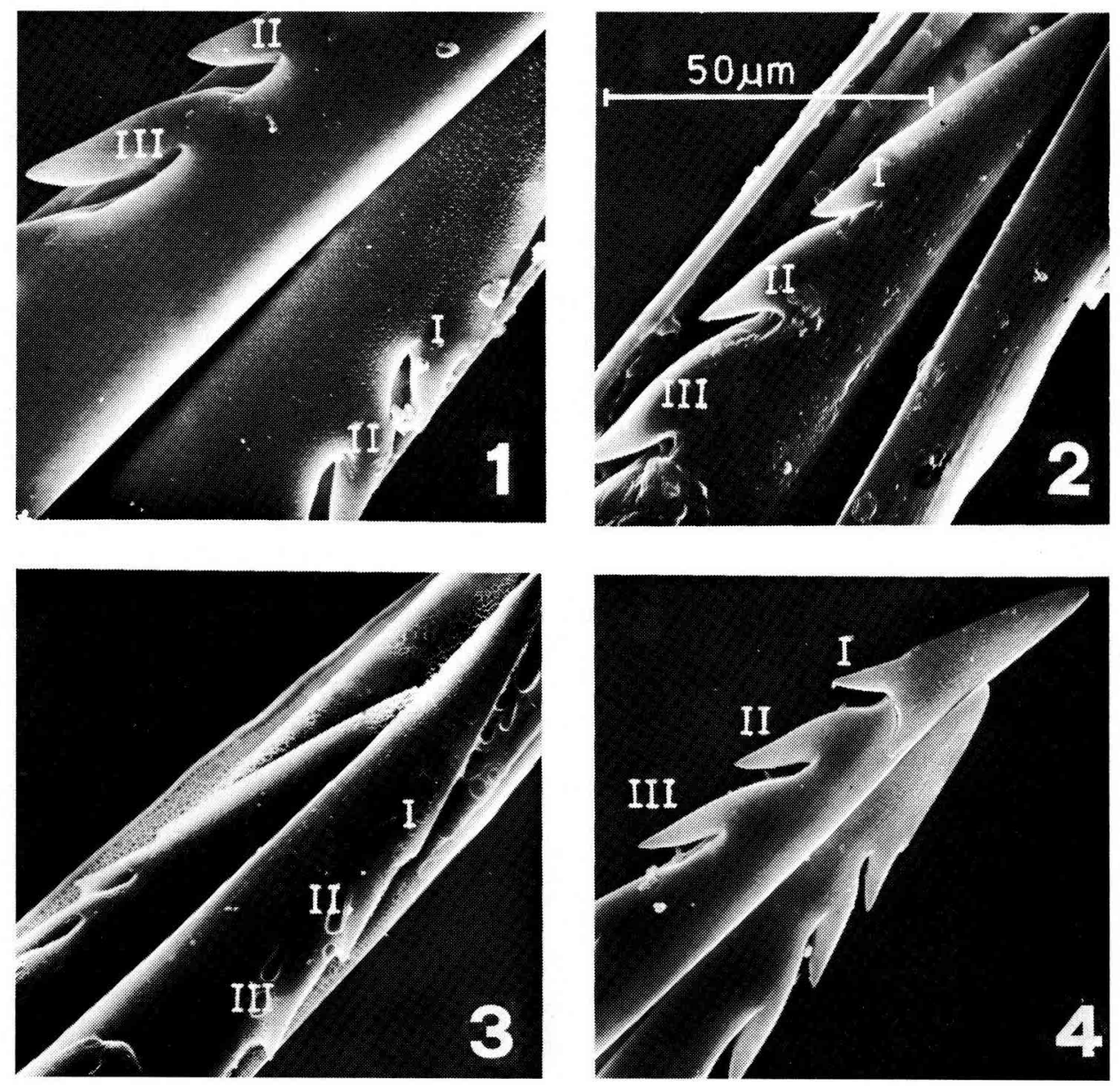

ABB. 6. - Stechborstenspitze der vier Apis-Arten mit den Widerhaken I bis III (REM): 1. A. dorsata. 2. A. mellifica. 3. A. cerana. 4. A. florea.

FIG. 6. - Pointe des soies barbelées chez les 4 espèces d'Apis avec leurs barbes I à III. (microscopie électronique à balayage).

6.1. A. dorsata. 6.2. A. mellifica. 6.3. A. cerana. 6.4. A. florea. 


\section{B. 2. - Neunter Widerhaken}

Zum weiteren interspezifischen Vergleich wurde der Winkel ausgewertet, den der (willkürlich ausgewählte) neunte Stechborstenwiderhaken zur Stechborstenlängsachse einnimmt : Mit $20^{\circ}$ ist er bei $A$. dorsata von allen vier Arten and weitesten von der Längsachse abgespreizt $(\mathrm{s}=1,49)$, es folgen A. florea mit $18^{\circ}(\mathrm{s}=1,15)$, A. mellifica mit $10^{\circ}(\mathrm{s}=0,94)$ und $A$. cerana mit $7^{\circ}(\mathrm{s}=0,94)$.

Zur Ermittlung der Einschnittiefe des neunten Widerhakens wurde die Gerade vom tiefsten Punkt des Einschnittes bis zur Widerhakenspitze vermessen : Wie Abb. 7 belegt, weist der neunte Stechborstenwiderhaken von $A$. dorsata den tiefsten Einschnitt auf, es folgen $A$. mellifica, A. florea und $A$. cerana (siehe auch Abb. 8).

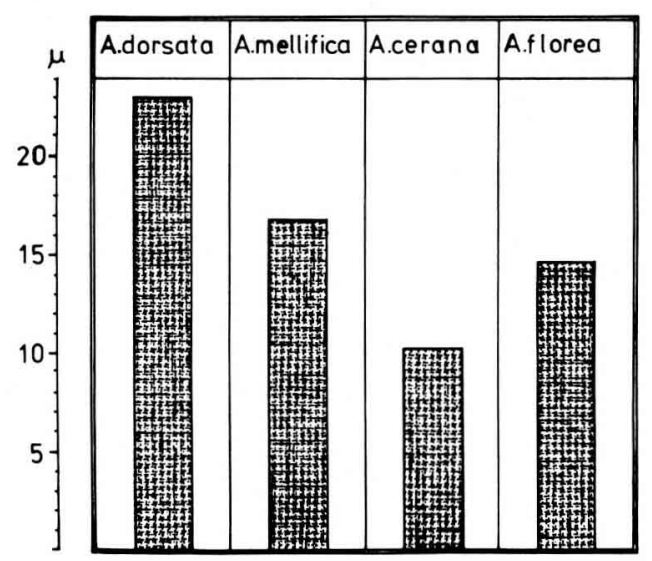

Aвв. 7. - Posteriore Einschnittiefe des IX. Stechborstenwiderhakens der vier Apis-Arten. Angaben in Mikrometer.

FıG. 7. - Profondeur des crans postérieurs de la $9^{e}$ barbe des soies barbelées des 4 espèces d'Apis. Mesures en $\mu \mathrm{m}$. 

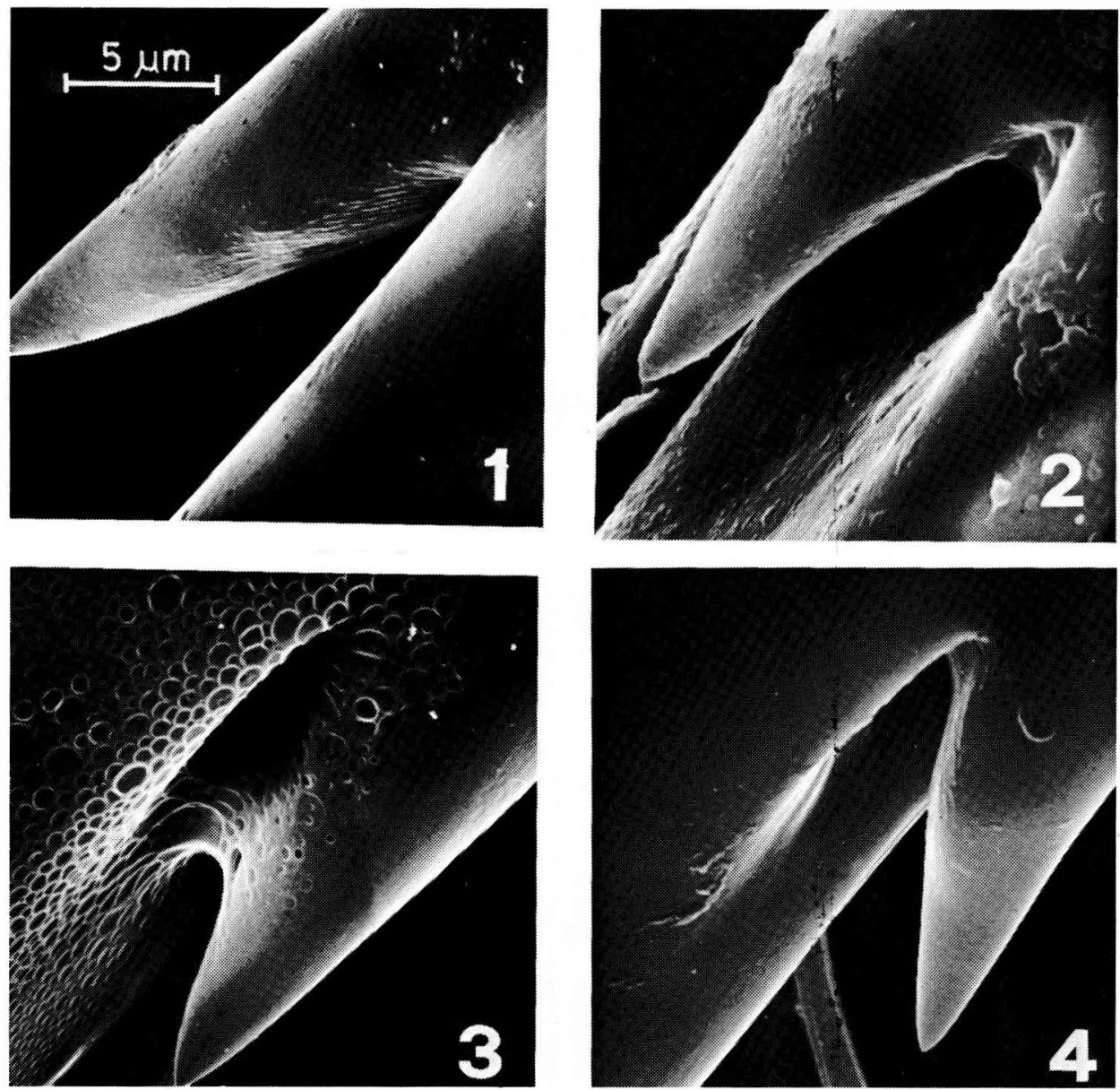

Авв. 8. - Der IX. Stechborstenwiderhaken der vier Apis-Arten (REM):

1. A. dorsata. 2. A. mellifica. 3. A. cerana. 4. A. florea.

FIG. 8. - La $9^{e}$ barbe des soies barbelées chez les 4 espèces d'Apis (microscopie électronique à balayage). 8.1. A. dorsata. 8.2. A. mellifica. 8.3. A. cerana. 8.4. A. florea.

\section{IV. - DISKUSSION}

Die Entwicklung langer Widerhaken, die zur Schaftlängsachse einen ausgeprägten Winkel bilden, befähigt die Honigbienen, ihren Stachel fest im Opfer zu verankern. Zusammen mit der - bei $A$. mellifica stark zurückgebildeten - Muskulatur zwischen Stachelapparat und Tracheenplatten führt dies zu häufigerer Autotomie (RIETSCHEL, 
1955) und erhöht gleichermassen die Ëffizienz des Stachelapparates (HermanN, 1971) : Je tiefer ein Stachel z.B. in die Lederhaut eines Warmblüters eindringt, desto grösser ist einmal der Schmerz infolge der mechanischen Vorgänge; zum anderen steigert die verlängerte und tiefere Giftinjektion die Schmerzempfindung. Schliesslich aber markiert die Emission der Alarmstoffe das gestochene Objekt, und der Stachelapparat als Träger der Alarmpheromone kann in der Regel nicht ohne Hilfsmittel entfernt werden.

Wie hier nicht im einzelnen aufgeführte Untersuchungen des Stachelapparates der vier Apis-Arten gezeigt haben, spiegeln eine Reihe verschiedener Parameter, wie z.B. Gesamtlänge des Schaftes, Länge des Widerhakenabschnittes, Länge, Breite und Dicke des Bulbus usw. in ihren Proportionen lediglich die äusseren Grössenverhältnisse der einzelnen Arten wider. Form und Grösse der so spezialisierten Widerhakenstrukturen haben jedoch ein abweichendes Bild ergeben : Die Widerhaken der Stechborsten sind bei $A$. cerana in allen untersuchten Punkten deutlich schwächer entwickelt als die der drei anderen Apis-Arten. Dieses Ergebnis stimmt überein mit den Angaben von SaKaGAMI und AKahiRa (1960), die bei einem Vergleich zwischen A. mellifica und $A$. cerana fanden, dass die Östliche Honigbiene ihren Stachel nach dem Einstich häufiger wieder aus dem gestochenen Objekt herausziehen kann als ihre westliche Schwesternart, dass sie somit seltener Autotomie zeigt. Von den genannten Autoren, die in der Ausbildung der Stechborstenwiderhaken keine Unterschiede zwischen den beiden Arten feststellen konnten, wurde dieses Phänomen ausschliesslich auf die geringere Reduzierung der Haltemuskeln zwischen Stachelapparat und Tracheenplatten zurückgeführt. Die oben beschriebenen Widerhakenvergleiche belegen jedoch, dass auch die mangelhafte Ausbildung der Stechborstenwiderhaken die seltener vorkommende Autotomie von A. cerana mitbedingt.

Nimmt man nun - entsprechend der Ausbildung der Widerhaken, und unter Berücksichtigung der äusseren Proportionen - eine Reihung innerhalb der Gattung vor, so steht $A$. florea an der Spitze, es folgen $A$.dorsata, A. mellifica und A. cerana. Die hervorragende Position von $A$. dorsata und $A$.florea erhält einen besonderen Stellenwert durch neueste Befunde (Veith, WeIss und Koeniger, 1977, im Druck), wonach diese beiden freibrütenden Arten neben dem - allen vier Arten gemeinsamen flüchtigen Alarmstoff Isopentylacetat eine weitere Alarmsubstanz mit deutlich geringerem Dampfdruck im Stachelapparat haben.

Diese Verbindung (2-Decen-1-yl-acetat) dient nach unseren Beobachtungen dazu, gestochene Feindobjekte zu markieren. Die gut entwickelten Widerhakenstrukturen der freibrütenden Arten gewährleisten dabei eine hohe Autotomierate und damit eine effektive Feindmarkierung.

Nach den Forderungen von RiETsChel (1955) und HERMANN (1971) bedeutet dies, dass $A$. dorsata und $A$. florea im Hinblick auf die hier geprüften Kriterien in ihrer Kolonieverteidigung einen höheren Grad der Spezialisierung erreicht haben als A. mellifica und A. cerana. Angesichts der ungeschützten Lebensweise der 
freibrütenden Arten könnte dies einen grösseren Vorteil für die Selektion bedeutet haben, als das bei höhlenbrütenden Arten der Fall gewesen wäre.

Das oben angesprochene Spezialisierungsgefälle stimmt nicht mit der herkömmlichen Auffassung der Evolution innerhalb der Gattung Apis überein : Nach Art von Kommunikation, Wabenbau usw. wird $A$. florea als primitiv und $A$. mellifica als hochentwickelt bezeichnet (LINDAUER, 1956).

Einen weiteren Widerspruch zu dieser These ergaben in jüngerer Zeit Untersuchungen von RUTTNER (1975), wonach der metatarsale Haftapparat von A.florea am stärksten abgeleitet ist, und der von $A$. cerana am ursprünglichsten gestaltet ist (siehe auch KoENIGER, 1976).

Die oben beschriebenen Ergebnisse zeigen übereinstimmend, dass A. florea im Bereich zweier funktionell völlig unterschiedlicher morphologischer Strukturen als die am stärksten abgeleitete Art der Gattung Apis anzusehen ist, A. cerana erscheint im Bezug auf die untersuchten Strukturen als die ursprünglichste Art. Nun ist nach diesen Befunden eine neue Reihung innerhalb der Gattung zwar verfrüht; jedoch wird deutlich, dass die Evolution innerhalb einer Gattung nicht nach einem starren, linearen System verläuft, sondern dass sie vielmehr ein differenzierter Prozess ist mit unterschiedlichen spezifischen Richtungen.

\section{v. - DANKSAGUNG}

Diese Arbeit wurde mit Unterstützung der Graduiertenförderung der Johann Wolfgang Goethe Universität in Frankfurt/M durchgeführt. Herrn Doz. Dr. KoENIGER sowie Herrn Prof. Dr. F. RutTNER bin ich für die kritische Durchsicht des Manuskriptes verbunden.

\section{VI. - LITERATUR}

BÄHRMANN R., 1967. - Vergleichend-morphologische Untersuchungen an Arbeitsbienen und Drohnen von Apis cerana F. Z. Bienenforsch., 9 (1), 32-46.

HERMANN H. R., 1971. - Sting autotomy, a defensive mechanism in certain social Hymenoptera. Insectes soc., $18(2), 111-120$.

Koeniger N., 1976. - Neue Aspekte der Phylogenie innerhalb der Gattung Apis. Apidologie, 7 (4), 357 366.

Lindauer M., 1956. - Über die Verständigung bei indischen Bienen. Z. vergl. Physiol., 38, 521-557.

RIETSChel P., 1955. - Was ist Autotomie? Umschau, 55, 174-176.

RutTNer F., 1975. - Ein metatarsaler Haftapparat bei den Drohnen der Gattung Apis (Hymenoptera : Apidae). Entomologica Germanica, 2 (1), 022-029.

SAKagami S. F., AKahiRa Y., 1960. - Studies on the Japanese honeybee, Apis cerana cerana Fabr. VII. Two opposing adaptations in the poststinging behaviour of honeybees. Evolution, 14 (1), 29-40.

Snodgrass R. E., 1956. - Anatomy of the Honey Bee. Comstock Publishing Associates Ithaca, New York. 
VEITH H. J., WeISS J. und KoENIGER N., 1977. - A new alarm pheromone (2-decen-1-yl-acetate) isolated from stings of Apis dorsala and Apis florea (Hymenoptera : Apidae) Experientia (i. Druck).

ZANDER E., 1951. - Der Bau der Biene. Aus : Handbuch der Bienenkunde in Einzeldarstellungen. Eugen Ulmer, Stuttgart.

\section{RÉSUMÉ}

1. Il résulte de la comparaison interspécifique de la morphologie de l'appareil venimeux chez les 4 espèces d'Apis (Apis dorsata Fabricius, 1973, A. mellifica Linnaeus, 1758, A cerana Fabricius, 1793, A. florea FABricius, 1787) que la majeure partie des structures correspondent par leurs dimensions aux proportions extérieures des espèces respectives. Les barbes spécialisées des gaines de l'aiguillon et des soies barbelées sont dans une situation divergente.

2. Barbes des gaines de l'aiguillon : A. florea vient en ête avec 4 paires de barbes bien développées, puis suivent $A$. dorsata et $A$. cerana; $A$. mellifica ne montre en général aucune barbe possédant de cran postérieur mesurable.

3. Barbes des soies barbelées : A. dorsata, A. mellifica et $A$. cerana possèdent 11 barbes sur chaque soie; A. florea seulement 10.

Sur les soies barbelées d'A. dorsata, $A$. mellifica, et $A$. florea les trois premières barbes sont bien formées; chez $A$. cerana ces structures sont très faiblement développées.

4. Neuvième barbe des soies barbelées : l'angle entre la $9^{c}$ barbe et l'axe longitudinal de la soie barbelée a les valeurs suivantes: A. dorsata: $20^{\circ}(\mathrm{s}=1,49)$, A. florea : $18^{\circ}(\mathrm{s}=1,15)$, A. mellifica : $10^{\circ}$ $(\mathrm{s}=0,94)$ et $A$. cerana : $7^{\circ}(\mathrm{s}=0,94)$.

On obtient la séquence suivante pour la profondeur du cran postérieur : $A$. dorsata : 22,95 $\mu \mathrm{m}$ $(\mathrm{s}=1,52)$, A. mellifica $: 16,75 \mu \mathrm{m}(\mathrm{s}=0,64)$, A. florea $: 14,62 \mu \mathrm{m}(\mathrm{s}=0,7)$ et A. cerana $: 10,13 \mu \mathrm{m}$ $(\mathrm{s}=0,31)$.

5. D'après les proportions indiquées ci-dessus pour les quatre espèces, les barbes d'A. florea sont les mieux développées pour tous les critères étudiés; viennent ensuite $A$. dorsata et $A$. mellifica, les structures sont le plus faiblement développées chez $A$. cerana qui, par conséquent, semble être l'espèce la plus primitive. 